\title{
ColdFront: Resource Allocation Management System
}

\author{
Andrew E. Bruno \\ aebruno2@buffalo.edu \\ Center for Computational Research, University at Buffalo \\ Buffalo, New York, USA
}

\begin{abstract}
ColdFront is an open source resource allocation management system, designed to provide a central portal for administration, reporting, and measuring scientific impact of cyberinfrastructure resources. It enables managing access to a diverse set of resource types across large groups of users and provides a rich set of extensible meta data for comprehensive reporting and integration with legacy systems. In this paper we introduce ColdFront, describe three main stakeholders, present the features and integrations currently implemented, and make the case for why ColdFront is vital to enabling research and reducing time-to-science. ColdFront is freely available at https://github.com/ubccr/coldfront.
\end{abstract}

\section{CCS CONCEPTS}

- Computer systems organization; • Information systems $\rightarrow$ Storage management; $\bullet$ Social and professional topics $\rightarrow \mathrm{Com}$ puting / technology policy; Computing equipment management;

\section{KEYWORDS}

HPC administration, systems management, allocations, storage, linux clusters

\section{ACM Reference Format:}

Andrew E. Bruno and Doris J. Sajdak. 2021. ColdFront: Resource Allocation Management System. In Practice and Experience in Advanced Research Computing (PEARC '21), July 18-22, 2021, Boston, MA, USA. ACM, New York, NY, USA, 5 pages. https://doi.org/10.1145/3437359.3465585

\section{INTRODUCTION}

Cyberinfrastructure (CI) centers encompass a diverse portfolio of shared resources providing the backbone of numerous scientific research initiatives. Effectively and efficiently managing access to these resources is imperative for campus CI support staff. For many, this is accomplished using home grown solutions that can be costly to develop, maintain and improve. A dedicated staff member is typically needed to manage this process which includes account management, access control, usage tracking, billing, and reporting. Managing these processes is burdensome and consumes valuable staff resources that can be better utilized elsewhere. Having a customizable, easy to use, and automated framework to manage not only the library of available resources, but also user access to those

This work is licensed under a Creative Commons

Attribution-NonCommercial-ShareAlike International 4.0 License.

PEARC '21, July 18-22, 2021, Boston, MA, USA

(C) 2021 Copyright held by the owner/author(s)

ACM ISBN 978-1-4503-8292-2/21/07.

https://doi.org/10.1145/3437359.3465585

\author{
Doris J. Sajdak \\ djm29@buffalo.edu
}

Center for Computational Research, University at Buffalo Buffalo, New York, USA

resources, enhances campus CI security, frees up valuable staff resources, improves management's reporting capability, and ensures a simplified process for campus $\mathrm{CI}$ end users. To our knowledge, no commercial or open source software packages exist to fill this need prompting us to build ColdFront, an open source framework that provides a central web-based portal and back-end infrastructure for administration of shared resources, allocation management, and collecting data useful for determining the impact of campus CI resources. Throughout the paper we use the term cyberinfrastructure (CI), as used by many funding agencies like NSF [8], for referring to research focused environments that support advanced data acquisition, storage, management, mining, visualization and other computing and information processing services.

ColdFront is currently being developed and run in production at the Center for Computational Research (CCR) at the University at Buffalo (UB). CCR provides a high performance computing (HPC) environment and on-premise cloud infrastructure supporting research across the university. CCR's compute resources [4] include three HPC clusters containing a heterogeneous array of hardware including over 1,500 nodes with over 32,500 processor cores, various flavors of GPUs, Infiniband, OmniPath, and Ethernet networks, a 2PB Pananas scratch system, and a 1.5PB Vast flash storage system. Five system administrators support this infrastructure that provides more than $1 \mathrm{PFlop} / \mathrm{s}$ of peak performance compute capacity. Also supported are file servers, databases, restricted software licenses, and research-specific servers and websites for research labs at the university. CCR provides access to these resources to all UB faculty, students, and staff as well as local industry business partners supported by the university's economic development office. At any given time there are over 1,000 active users of various resources supported by CCR.

Managing the allocations to these shared resources has traditionally been a manual process for the CCR CI staff similar to the approach used by many academic CI centers. Any commercial software that exists is either specific to a hardware product or cost prohibitive for academic HPC centers that are often budget constrained. We were unable to find any open source software to address this need and found upon surveying staff at other HPC centers that the majority use homegrown scripts and processes. Developing and maintaining in-house tools to accomplish this task consumes valuable, often scarce, staff resources. A flexible framework for managing access to limited shared resources via allocations, collecting scientific impact data such as grants and publications, and providing reporting capabilities is an unmet need in the scientific computing community. To address this gap, CCR has begun development of ColdFront, an open source system for managing user allocations to shared resources. ColdFront, specifically tailored for $\mathrm{CI}$ centers, is designed to manage allocations for a diverse set of resources including HPC clusters, co-located departmental and lab 
servers, software licenses, digital storage, scientific instrumentation, cloud subscriptions, and data access requests. It is designed to complement existing tools, such as Slurm and OpenStack, which manage access to individual hardware and software components. Though every institution will have its own policies for granting access to a resource, how that access gets provided is nearly universal. Through the flexibility of ColdFront's plug-ins, access policies can be implemented easily to automate the back-end systems resulting in significant cost savings for campuses both small and large.

In this paper we describe three main stakeholders, provide a highlevel overview of the ColdFront architecture, showcase examples of how ColdFront can be used to manage access to CI resources, and our plans to develop ColdFront into the de facto open source platform for CI resource and access management.

\section{STAKEHOLDERS}

Three groups of stakeholders were targeted for the initial design of ColdFront: principal investigators (PIs) and CI resource end users, $\mathrm{CI}$ center directors and other decision makers, and CI center system administrators. While each group has its own set of unique needs in this process, many requirements are shared by all stakeholders. Center directors and decision makers need a mechanism for gathering updated and current research information from PIs, an easy process for the review and approval of access requests for their center's resources, and unified reporting capabilities. Principal investigators require an easy way to provide the information about their research such as grant, publication, and project information that the center is requesting. They will also benefit from a simple process for viewing what resources are available to them and then requesting access to resources for their group. Once granted access to a resource via an allocation, PIs and their group members should be able to view the current state and usage level of their allocation. CI center system administrators require operational support to manage resources and the integrations between them, manage and process requests for access, and automate system tasks such as account creation and user access control.

While individual tools exist for account management and access control, job scheduling and resource usage, and resource tracking, no tools exist that integrate all of these into a single ecosystem to allow cross-referencing and federation of this information. While there are tools that exist to provide system administration staff with the ability to manage an allocations process, none were found that integrate allocations management, usage reporting, and automatic management of user access to resources. The few options available are either tied directly to a single resource manager, such as Slurm Bank [5], or have not been updated in over 10 years in the case of Gold [7], making it unusable for most modern CI centers. The Gold Allocation manager only supports HPC clusters, not other hardware resources such as storage and instrumentation or nonphysical resources such as databases and software licenses. This is used to keep track of how much is in your allocation "wallet" or "bank." The closest tool to what ColdFront provides for allocations management is the XSEDE Resource Allocation System (XRAS) [3]. While XRAS supports the submission, review, and administration of allocation requests via a well-defined allocation and review process, it is oriented more towards sites affiliated with XSEDE and does not integrate with other tools to manage access to resources. Management of the physical resources is left to individual XSEDE sites. Having a single flexible tool to manage all of this for all stakeholders was a primary goal in the development of ColdFront.

\subsection{System Staff}

CI system administrators and staff can use ColdFront as a management portal and command line tool to approve/deny resources allocation requests and define when allocations expire. Resources can be public or private based on a per-user or per-group basis. Custom attributes can be defined on allocations to enable automated access control and integration with external applications such as job schedulers and billing systems. ColdFront provides a unique feature for requiring PIs to periodically review their projects to ensure user access is kept up to date which helps keep systems secure and data protected. Additionally, the annual project review allows CI staff to capture important meta data such as grants and publications to help measure the center's impact. ColdFront integrates with multiple authentication backends such as LDAP and OAuth2/OpenID connect which eases the deployment into existing $\mathrm{CI}$ infrastructure.

\subsection{Center Directors}

CI center directors can use ColdFront to gain important insight into the operations of the center. They can review, approve/deny allocations requests and explore project data (users, publications, grants, research output). ColdFront enables directors to easily interact with PIs through the project review process and ensure adequate justification is provided for using the center's resources. Most importantly, directors can measure center impact based on allocation data such as grants, publications, and other research output providing return on investment (RIO) metrics that help to position centers for sustainability.

\subsection{Researchers (PIs) and Users of the Resources}

Principal investigators (PIs) and their research groups can use ColdFront as a self-service portal for requesting access to center resources. They can add/remove user access to/from allocated resources without requiring system administrator interaction. Individual users of the group can be selected for elevated "manager" status allowing them to handle tasks on behalf of the PI such as requesting new allocations, adding grants and publications, and even adding/removing users. PIs can monitor their resource utilization such as storage and cloud usage. To obtain an allocation, PIs can submit information such as grants, publications, and other reportable data as justification which can be used for periodic review by center staff/allocation committees to demonstrate need for the resources. ColdFront sends out email notifications for expiring/renewing access to resources as well as notifications when allocations change status providing important information directly to the PIs.

\section{COLDFRONT ARCHITECTURE}

ColdFront is written in Python using the Django web framework [1]. The Django web framework organizes related sets of features 
into apps. Apps are combined together to make up a project or system. ColdFront separates its apps into two categories: core and plugins. The core apps support the core functionality of ColdFront. The plugin apps extend or modify the core functionality of ColdFront. For instance, plugins can integrate with third party systems for job scheduler account automation and access control. ColdFront currently integrates with third-party vendor software such as FreeIPA, OpenID Connect, OpenStack, Slurm resource manager, Storage APIs for quota reporting, Open OnDemand [6], and Open $\mathrm{XDMoD}$ [9]. The existing Django apps can be used as models to easily customize ColdFront to fit any CI center's use cases and policies. ColdFront supports multiple database backends including MySQL/MariaDB, PostgreSQL, and SQLite. Complete documentation for installing, configuration, and using ColdFront can be found at https://coldfront.readthedocs.io. ColdFront is also included in the HPC Toolset Tutorial [2] presented at past workshops for PEARC and Gateways conferences. The tutorial creates a fully functional virtual data center using docker and demonstrates how three open source applications, OnDemand, Open XDMoD, and ColdFront work in concert to provide core services for high performance computing centers.

\section{USE CASES}

Currently at CCR, all access to resources must be done using ColdFront. All faculty are required to maintain at least one project and access to the resources they want to use is done through allocations. If allocations are not renewed before they expire, access to those resources is removed for the group. Faculty are required to complete an annual project review to ensure all reportable data provided is up-to-date. They're asked to remove any users who are no longer working in their group, as well as update the project description if their work has changed. This process takes 5-10 minutes once a year. These are some of the ways we use ColdFront at CCR and exemplify real world production use cases:

\subsection{Allocations to Resources}

A principal investigator would like access to the HPC cluster resource for her research group. She logs into ColdFront and creates a project that informs the center staff about her research. The PI then adds all of her students and collaborators to the project. She is able to review in a drop down list all of the center's resources that she is permitted to request access to and selects the HPC cluster resource. She provides a justification for the request and selects all or a subset of her project users to add to the allocation. She might then also request allocations to other resources such as storage, a software license or the on-premise cloud. Feedback can be provided to the PI within ColdFront so there is always a single location to view and track all information regarding the allocation. Once a decision is made by center management and/or system administrators regarding the allocation, the status of the allocation is changed in ColdFront for the PI to view. The PI and group users are notified via email and access to the cluster is automatically granted via the Slurm plugin.

\subsection{Automated Access Control}

System administrators want to automate access to a campus HPC cluster running the Slurm job scheduler. A request has come in for an allocation on the HPC cluster. A system administrator associates the allocation request with additional attributes required by the Slurm resource manager (i.e. account name, group name, cluster name, limits). These attributes are fed to Slurm using the ColdFront plugin and this allows the creation of a Slurm account, associations, and any specified limits. Once these are set and the allocation is marked "active" the ColdFront Slurm plug-in makes the changes and the PI and group members are granted access to this resource. When the allocation expires or is expended, the next time the Slurm plug-in runs, the group's access to the resource is removed.

\subsection{Measuring Scientific Impact}

The HPC center director needs to report on scientific impact/return on investment to institutional administrators. When it's time to prepare his annual report on the center's usage and impact to the institution, the center director turns to the combination of ColdFront and Open XDMoD [9] data to provide quantifiable data. He requires all of the PIs who use the center's resources to update their project information annually in ColdFront so that he can accurately report on the fields of science the center supports, publications that have been made possible by access to the center's resources, and supporting grants funded, in part, by the availability of access to these resources. He then utilizes Open XDMoD to provide detailed usage information for the last year which he can attribute to specific departments and research groups. This combination of information provides a detailed picture that helps define the impact of the campus research computing infrastructure. These reports are also useful when planning the next purchase of storage or compute resources. With this combination of data from ColdFront and Open $\mathrm{XDMoD}$, he can see how the resources (compute job sizes, storage usage, GPU utilization, etc.) are currently being utilized and make informed decisions for new technology purchases.

\subsection{Managing Diverse Resource Types}

The center allows for PIs to purchase their own equipment or buy time on shared equipment, known as "condo" computing. The resources are setup in ColdFront to allow private access based on accounts or groups. When the PI goes to request an allocation for this type of resource he/she only sees the resources they have purchased. System administrators configure the resource in ColdFront with the proper job scheduler attributes so that users on the allocation are given the proper access to only the hardware purchased by the PI. ColdFront's flexibility allows for detailed and targeted configurations that were not possible before.

Tracking requests for data sets. Various projects on campus may generate data sets that are useful to members of the research community. Rather than store multiple copies of data, which is a waste of an expensive and limited storage resource, it would be more efficient to provide a shared location for the data and make it available to interested researchers. In order to advertise the datasets, as well as track and report on how the data is being used, these data sets are added to ColdFront as resources and researchers may request access to them. By configuring ColdFront to require a brief 
description of the intended use of the data, we can track and report on how this data is being utilized.

Providing access to restricted software licenses. CCR uses ColdFront to configure software licenses purchased by the center or by a PI. The access to the software on the system is accomplished using unix groups. We configure ColdFront to contain the software information for PIs to find in their drop down menu of resources and the end-user license agreement (EULA) they must agree to (if applicable). If the software is meant for a restricted group of people, we configure ColdFront so the resource does not display on the drop down list unless the user is in the allowed group. Anytime the allocation is renewed, the PI must agree again to the EULA. Being able to track this information is valuable to center staff who need to prove the center is compliant with software terms. PIs find the flexibility of ColdFront's allocations management helpful when managing access to limited resources. If they have a limited number of seats for a license, for example, they are able to select a subset of users on their project to add to the allocation thus decreasing contention for the license.

While CCR uses ColdFront for access to all resources in our data center, there are many organizations using ColdFront to manage parts of their infrastructure. Some use it for new clusters they've put into production recently; others are using it just for one type of resource such as storage. We feel it would be extremely useful to managing shared scientific laboratory equipment and are interested in testing this use case in the future.

\section{CONCLUSIONS AND FUTURE WORK}

CI resources are vital for scientific research but the day-to-day grind and significant personnel investment of managing these resources is often overlooked and taken for granted. Much effort is spent on how scientific research will utilize various resource types, the software applications involved, and computational needs required to produce outcomes. Rarely mentioned are the systems required by CI centers to effectively and securely manage access to these resources which are often times being utilized by heterogeneous groups of researchers. The administrative systems run by CI centers don't directly produce flashy scientific outcomes but provide the infrastructure backbone to support important scientific research. Without effective tools to administer CI centers, time-to-science suffers and accessing resources becomes the bottle neck.

$\mathrm{CI}$ centers are charged with managing an ever increasing number of diverse resources that require significant effort to not only install and maintain but to effectively manage and coordinate access. In addition to traditional local on-premise compute and storage resources more institutions are utilizing public and private cloud platforms. Allocations to the cloud tend to have more advanced requirements and integrating these types of resources into the library of options available to researchers is paramount. External resources also include national $\mathrm{CI}$ resources made available, for example, through XSEDE [10], but often researchers are unaware of them at the local level or the access to these resources is viewed as daunting. Disseminating the information to end users on what external resources are available, how they differ from on-premise resources and the processes for gaining access to them can be full time job for a campus CI research facilitator. Having to deal with multiple entry points, portals, processes, and other account or reporting tasks is frustrating and often discouraging enough to repel the user. A single location for a researcher to view the myriad options of available resources, request access to the desired resources, and then launch the login to those resources will provide a more streamlined experience for them and help to prevent complexity attrition. A single unified system that's available to CI centers with the source code freely available and community driven by contributions and improvements is paramount for the successful management of these resources for all stakeholders involved.

ColdFront aims to fill this void with bold ambitions of being the de facto software for managing CI resources. It bridges the gap between commercially available systems that might be unaffordable and inflexible and existing homegrown processes currently running at many CI centers. ColdFront's flexible architecture allows for the integration of existing services that may already be deployed at a CI center making it ideal for updating and improving current allocations workflows without having to reconstruct the whole process. ColdFront is released under the GPLv3 license and available at https://github.com/ubccr/coldfront.

ColdFront is a relatively new project under heavy development. Based on feedback from the community we plan to enhance the allocation review processes and allow more flexibility and configuration for centers to incorporate their own policies and procedures. The invoicing feature of ColdFront is currently the least developed and we plan on making improvements to this. The community has requested various features to enhance the billing processes and allow for automated pricing on allocations.

The more robust systems we have in place for managing CI resources the better we can serve the scientific community and improve time-to-science which has an overall net positive impact on scientific outcomes.

\section{ACKNOWLEDGMENTS}

This material is supported by resources at the Center for Computational Research (https://buffalo.edu/ccr) at the University at Buffalo. Partial support from NSF OAC 1541215.

\section{REFERENCES}

[1] Django Authors. 2021. Django. https://www.djangoproject.com. Online; accessed: 2021-04-05.

[2] HPC Toolset Tutorial Authors. 2021. HPC Toolset Tutorial. https://github.com/ ubccr/hpc-toolset-tutorial. Online; accessed: 2021-04-05.

[3] XRAS Authors. 2020. XSEDE Resource Allocations System. https://portal.xsede. org/allocations/about-xras. Online; accessed: 2021-04-05.

[4] Center for Computational Research, University at Buffalo. 2020. UB CCR Support Portfolio. http://hdl.handle.net/10477/79221.

[5] Paddy Doyle and Jimmy Tang. 2012. SLURM Bank, a collection of wrapper scripts to give slurm GOLD like capabilities for managing resources. https: //github.com/jcftang/slurm-bank. Online; accessed: 2021-04-05.

[6] Dave Hudak, Doug Johnson, Alan Chalker, Jeremy Nicklas, Eric Franz, Trey Dockendorf, and Brian L. McMichael. 2018. Open OnDemand: A web-based client portal for HPC centers. Journal of Open Source Software 3, 25 (2018), 622. https://doi.org/10.21105/joss.00622

[7] Scott Jackson. 2003. Gold Allocation Manager. https://github.com/anderbubble/ gold. Online; accessed: 2021-04-05.

[8] Office of Advanced Cyberinfrastructure. 2021. Office of Advanced Cyberinfrastructure (OAC). https://www.nsf.gov/cise/oac/about.jsp. Online; accessed: 2021-04-05.

[9] J. T. Palmer, S. M. Gallo, T. R. Furlani, M. D. Jones, R. L. DeLeon, J. P. White, N. Simakov, A. K. Patra, J. Sperhac, T. Yearke, R. Rathsam, M. Innus, C. D. Cornelius, J. C. Browne, W. L. Barth, and R. T. Evans. 2015. Open XDMoD: A Tool for the Comprehensive Management of High-Performance Computing Resources. 
Computing in Science Engineering 17, 4 (2015), 52-62. https://doi.org/10.1109/ MCSE.2015.68

[10] J. Towns, T. Cockerill, M. Dahan, I. Foster, K. Gaither, A. Grimshaw, V. Hazlewood, S. Lathrop, D. Lifka, G. D. Peterson, R. Roskies, J. R. Scott, and N. Wilkins-Diehr.
2014. XSEDE: Accelerating Scientific Discovery. Computing in Science Engineering 16, 5 (2014), 62-74. https://doi.org/10.1109/MCSE.2014.80 